\title{
Western Kenyan Anopheles gambiae showing intense permethrin resistance harbour distinct microbiota
}

\author{
Diana Omoke ${ }^{1,2}$, Mathew Kipsum² ${ }^{2}$ Samson Otieno², Edward Esalimba², Mili Sheth ${ }^{3}$, Audrey Lenhart ${ }^{4}$ (D), \\ Ezekiel Mugendi Njeru ${ }^{1}$ (10, Eric Ochomo ${ }^{2}$ (i) and Nsa Dada ${ }^{5,6^{*}}$ (1)
}

\begin{abstract}
Background: Insecticide resistance poses a growing challenge to malaria vector control in Kenya and around the world. Following evidence of associations between the mosquito microbiota and insecticide resistance, the microbiota of Anopheles gambiae sensu stricto (s.s.) from Tulukuyi village, Bungoma, Kenya, with differing permethrin resistance profiles were comparatively characterized.
\end{abstract}

Methods: Using the CDC bottle bioassay, 133 2-3 day-old, virgin, non-blood fed female $F_{1}$ progeny of field-caught An. gambiae s.s. were exposed to five times $(107.5 \mu \mathrm{g} / \mathrm{ml})$ the discriminating dose of permethrin. Post bioassay, 50 resistant and 50 susceptible mosquitoes were subsequently screened for $k d r$ East and West mutations, and individually processed for microbial analysis using high throughput sequencing targeting the universal bacterial and archaeal 165 rRNA gene.

Results: $47 \%$ of the samples tested $(n=133)$ were resistant, and of the 100 selected for further processing, $99 \%$ were positive for $k d r$ East and $1 \%$ for $k d r$ West. Overall, 84 bacterial taxa were detected across all mosquito samples, with 36 of these shared between resistant and susceptible mosquitoes. A total of 20 bacterial taxa were unique to the resistant mosquitoes and 28 were unique to the susceptible mosquitoes. There were significant differences in bacterial composition between resistant and susceptible individuals (PERMANOVA, pseudo- $F=2.33, P=0.001$ ), with presence of Sphingobacterium, Lysinibacillus and Streptococcus (all known pyrethroid-degrading taxa), and the radiotolerant Rubrobacter, being significantly associated with resistant mosquitoes. On the other hand, the presence of Myxococcus, was significantly associated with susceptible mosquitoes.

Conclusions: This is the first report of distinct microbiota in An. gambiae s.s. associated with intense pyrethroid resistance. The findings highlight differentially abundant bacterial taxa between resistant and susceptible mosquitoes, and further suggest a microbe-mediated mechanism of insecticide resistance in mosquitoes. These results also indicate fixation of the $k d r$ East mutation in this mosquito population, precluding further analysis of its associations with the mosquito microbiota, but presenting the hypothesis that any microbe-mediated mechanism of insecticide resistance would be likely of a metabolic nature. Overall, this study lays initial groundwork for understanding microbe-mediated mechanisms of insecticide resistance in African mosquito vectors of malaria, and potentially identifying novel microbial markers of insecticide resistance that could supplement existing vector surveillance tools.

\footnotetext{
*Correspondence: nsa.dada@nmbu.no; nsadada@yahoo.com

${ }^{5}$ Faculty of Science and Technology, Norwegian University of Life Science, Aas, Norway
}

Full list of author information is available at the end of the article

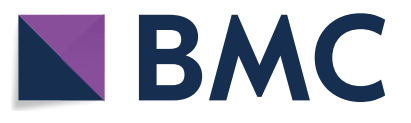

(c) The Author(s) 2021. This article is licensed under a Creative Commons Attribution 4.0 International License, which permits use, sharing, adaptation, distribution and reproduction in any medium or format, as long as you give appropriate credit to the original author(s) and the source, provide a link to the Creative Commons licence, and indicate if changes were made. The images or other third party material in this article are included in the article's Creative Commons licence, unless indicated otherwise in a credit line to the material. If material is not included in the article's Creative Commons licence and your intended use is not permitted by statutory regulation or exceeds the permitted use, you will need to obtain permission directly from the copyright holder. To view a copy of this licence, visit http://creativeco mmons.org/licenses/by/4.0/. The Creative Commons Public Domain Dedication waiver (http://creativecommons.org/publicdomain/ zero/1.0/) applies to the data made available in this article, unless otherwise stated in a credit line to the data. 
Keywords: Mosquito microbiota, Mosquito microbiome, Metabarcoding, Insecticide resistance, Anopheles gambiae s.S., $16 \mathrm{~S}$ rRNA gene amplicon sequencing, pyrethroid resistance

\section{Background}

Malaria remains an important global health problem, with $92 \%$ of all deaths occurring in Africa [1]. In Kenya, more than $70 \%$ of the population is at risk of the disease, with children aged $\leq 5$ years and pregnant women being the most vulnerable to infection [2]. The use of indoor residual spraying (IRS), long-lasting insecticidal nets (LLINs) and other interventions have led to measurable improvements in preventing malaria [3]. Continued reliance on insecticide-based interventions has also resulted in widespread insecticide resistance in malaria vectors, thus threatening malaria control efforts $[4,5]$. This is the case in western Kenya, where malaria vector control is increasingly being threatened by insecticide resistance due to selection pressure imposed by continued exposure to insecticides $[4,6]$.

Although insecticide resistance is increasingly prevalent [7], its underlying mechanisms are not fully understood. So far, four principal mechanisms of insecticide resistance have been described in mosquitoes, including: (i) metabolic resistance due to elevated activity of detoxification enzymes, (ii) target-site resistance due to genetic alterations at insecticide binding sites, (iii) cuticle modifications that prevent or reduce insecticide penetration, and (iv) behavioral changes resulting in avoidance of, or reduced contact with, insecticides [8]. Recent studies suggest that the mosquito microbiota may provide a fifth mechanism contributing to insecticide resistance $[9,10]$. Focusing largely on Anopheles albimanus across different geographical locations including Peru [9] and Guatemala [10], these studies have identified significant alterations of the mosquito microbiota associated with insecticide resistance, with enrichment of insecticide-degrading bacteria and enzymes in resistant mosquitoes [9].

The mosquito microbiota has been shown to affect mosquito physiology [11]. These microbes, which are predominantly acquired during the aquatic life stage from aquatic habitats, colonize mosquito tissues including the gut, reproductive tracts, exoskeleton, and haemocoel [12, 13]. Some of these microbes are beneficial to mosquitoes through their role in nutrient provisioning, immunity and development, and subsequent contributions to mosquito fitness [12]. They also help provide protection against pathogens by modifying the host's immune system or by synthesizing specific toxins [12]. The mosquito microbiota can influence and/or be influenced by several mosquito-related factors including mosquito species, developmental stage, genetics, and sex [11]. In mosquito vectors of malaria, the microbiota play important roles in malaria parasite development, survival, and sporozoite prevalence, thus modulating vector competence [14-17].

Recent studies on the effects of insecticide exposure on microbes associated with mosquitoes and their habitats have so far focused on Anopheles stephensi, Anopheles albimanus and Anopheles arabiensis [9, 10, 18, 19]. The microbiota of Anopheles gambiae sensu stricto (s.s.) has, however, largely been unexplored in relation to insecticide resistance. Of particular importance is pyrethroid resistance-a major concern in Kenya, where this class of insecticide is predominantly used in LLINs and IRS [6, 20, 21]. To address this research gap, this study characterized and compared microbiota between pyrethroid resistant and susceptible An. gambiae s.s. from an area with intense pyrethroid resistance in Western Kenya. Mosquitoes were also screened for gene mutations that mediate knockdown resistance $(k d r)$ to pyrethroids, in order to characterize any associations between the mosquito microbiota and $k d r$ genotype. We discuss these findings on An. gambiae s.s., and highlight their implications for insecticide resistance monitoring and management.

\section{Methods}

\section{Mosquito collections}

Mosquito collections were conducted in April and May 2018 in Tulukuyi village located in Bungoma County $0.56^{\circ} \mathrm{N} 34.56^{\circ} \mathrm{E} 1427 \mathrm{~m}$ ASL (Fig. 1). Previous studies conducted by Ochomo, et al. [20] indicated that An. gambiae s.s. was the most predominant species in Bungoma and had high resistance levels to pyrethroids. Sampling was performed by aspiration of blood fed and gravid mosquitoes from 39 houses. Mosquitoes were placed in labelled paper cups with information identifying the collection date and collection site. A piece of cotton wool soaked in $10 \% \mathrm{w} / \mathrm{v}$ sugar solution was placed on top of the netting material covering the paper cup to sustain the collected mosquitoes. The paper cups were then placed in a cool box and transported to the laboratory.

\section{Generation of $F_{1}$ progeny from field-collected mosquitoes}

Prior to species identification, forced oviposition was used to generate isofemale $F_{1}$ progeny from field collected blood-fed and/or gravid female mosquitoes. In this study, we focused on the $F_{1}$ generation (closest to the wild population) in order to standardize rearing conditions and physiological characteristics so as to 


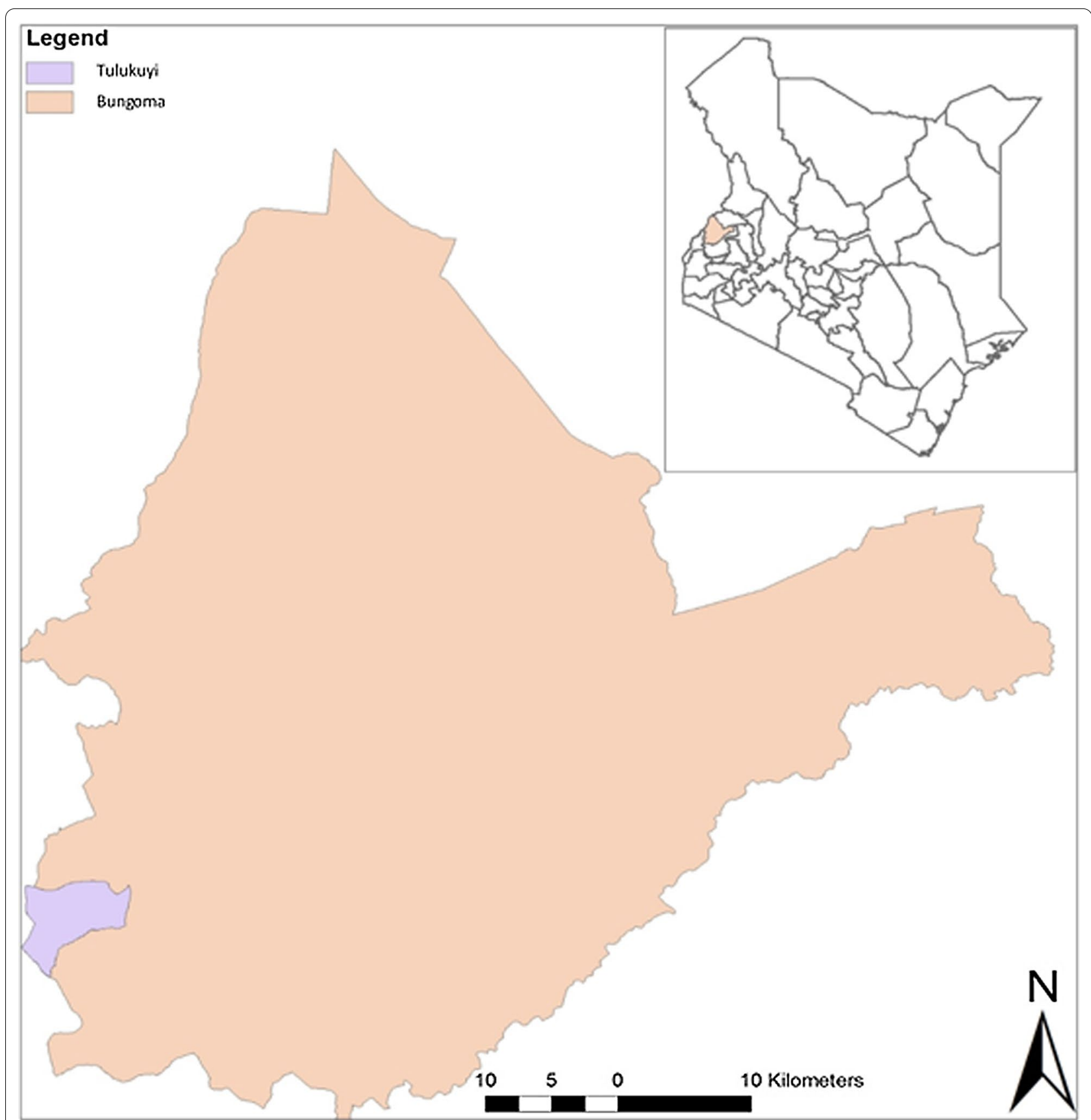

Fig. 1 Map of Kenya (Right) showing Bungoma County (in expanded view) where adult mosquito collections were conducted. Adult female Anopheles gambiae s.s. were collected from Tulukuyi village and $F_{1}$ progeny resulting from these mosquitoes were analysed

eliminate any confounding that not standardizing would have introduced-the wild-caught mosquitoes were only used to generate sufficient samples for this standardized design and were not processed beyond species identification. Individual mosquitoes were placed in separate $50 \mathrm{ml}$ falcon tubes containing damp cotton wool topped with filter paper for egg laying. Following egg laying, each adult female was transferred into individual $1.5 \mathrm{ml}$ reaction tubes for molecular species identification (described below). $F_{1}$ eggs from each isofemale were removed and placed into separate clean larval trays containing distilled water for hatching, while the parents underwent species identification as described below. Following species identification, all larvae from isofemales identified as 
An. gambiae s. s. were pooled, approximately 200 larvae per tray measuring $46 \mathrm{~cm}$ by $35 \mathrm{~cm}$ by $5 \mathrm{~cm}$ and reared together. Larvae were fed a combination of ground Brewer's yeast (Health Aid Company, Inc.), and Koi's choice premium fish food (Foster \& Smith, Inc. Rhinelander, WI) at a ratio of 1:2 for $A n$. gambiae until pupation. Using a dissecting microscope (Nikon C-PS, model no. $1,071,990)$, male and female pupae were separated within 24 hours of pupation in order to obtain virgin adult females. Female pupae were subsequently placed into cages for adult eclosion, while the males were euthanized and discarded. The resulting $F_{1}$ adult females $(\sim 377)$ were sustained on cotton balls soaked in $10 \%$ sugar solution for 2-3 days prior to insecticide susceptibility bioassays. In order to avoid potential confounding factors, all mosquitoes underwent identical standard handling and rearing in the insectary at the Kenya Medical Research Institute -Center for Global Health Research (KEMRICGHR), Kisian, Kisumu, under the following conditions: temperature of $27 \pm 2{ }^{\circ} \mathrm{C}$, relative humidity of $80 \pm 10 \%$, and photoperiod of 12:12 light: dark cycle.

\section{Molecular identification of mosquito species}

Using the ethanol precipitation method described by Collins, et al. [22], genomic DNA was extracted from whole individual field-collected female mosquitoes that were used to generate the $F_{1}$ progeny. $2 \mu \mathrm{l}$ of DNA from each individual, along with known An. gambiae s.s. DNA as positive control, were used as templates for the PCR reaction [23]. The reactions were performed using BIORAD thermal cycler model T100 under the following conditions: $95^{\circ} \mathrm{C}$ for $5 \mathrm{~min}$ followed by $95^{\circ} \mathrm{C}$ for $30 \mathrm{sec}$, $56^{\circ} \mathrm{C}$ for $30 \mathrm{sec}$ and $72^{\circ} \mathrm{C}$ for $30 \mathrm{sec}$ for 30 cycles, with a final extension at $72^{\circ} \mathrm{C}$ for $5 \mathrm{~min}$. Amplicons $(\sim 390 \mathrm{bp}$ for An. gambiae s.s.) were resolved by ethidium bromidestained agarose gel $2 \%$ electrophoresis.

\section{Permethrin resistance phenotyping}

A total of $133 \mathrm{~F}_{1}$ virgin, non-blood fed adult females aged 2-3 days were tested for permethrin resistance following the Centers for Disease Control and Prevention (CDC) guidelines for evaluating insecticide resistance [24]. The control bottle was coated with $1 \mathrm{ml}$ of acetone while the four test bottles were coated with $1 \mathrm{ml}$ of permethrin stock solution prepared with acetone, at a final concentration of $107.5 \mu \mathrm{g} / \mathrm{ml}(5 \times$ the dose for discriminating permethrin resistance in Anopheles). Anopheles gambiae Kisumu susceptible strain of the same age and physiological status were used to confirm the viability of the prepared bottles-all mosquitoes in the insecticide treated bottles died, while those in the acetone-treated bottles survived. Using the $F_{1}$ progeny, the bioassays were conducted for 30 minutes at the end of which permethrin resistance was recorded. Mosquitoes that were alive after the bioassay were categorized as resistant and subsequently killed by freezing, while those that were dead or moribund were categorized as susceptible. Phenotyped mosquitoes were immediately placed in $1.5 \mathrm{ml}$ Eppendorf tubes with unique identification codes and preserved at $-20^{\circ} \mathrm{C}$ for subsequent molecular processing. Mosquitoes from the control bottles were not further processed.

\section{Genomic DNA isolation and molecular processing DNA isolation and purification}

$250 \mu \mathrm{l}$ of $70 \%$ ethanol was added to each tube of individual $F_{1}$ mosquitoes and vortexed at high speed for $\sim 10$ seconds to surface sterilize the mosquitoes. This was followed by a vigorous rinse using a vortex mixer at high speed for $\sim 10$ seconds, then a gentle rinse by pipetting for $\sim 10$ seconds; each with $250 \mu$ l of nuclease free water. Genomic DNA from the whole mosquito was isolated and purified using the MasterPure ${ }^{\mathrm{TM}}$ Gram Positive DNA Purification Kit following the manufacturer's instructions (Epicentre Biotechnologies, Madison, USA). During DNA extraction, four blank controls (containing all the reagents used sans mosquito) and two $1 \mathrm{~g}$ soil samples from Kisian (as a distinct source of microbes) were also processed to catch any potential sample processing and cross contamination respectively. Purified DNA samples were stored at $-20^{\circ} \mathrm{C}$ for subsequent analysis.

\section{Detection of $k d r$-East and $k d r-W e s t$ alleles}

RT-PCR was used to detect the presence of both $k d r$-East and $k d r$-West alleles using DNA from $\mathrm{F}_{1}$ mosquitoes. Following the protocol by Bass, et al. [25], samples were processed using the MxPro-Mx3005P software 'Allele Discrimination-SNP's' program with $1.5 \mu \mathrm{l}(\geq 20 \mathrm{ng} / \mu \mathrm{l})$ of DNA as template. PCR was carried out under the following cycling conditions for $k d r$-East: $95^{\circ} \mathrm{C}$ for $10 \mathrm{~min}$ then 40 cycles of $95^{\circ} \mathrm{C}$ for 10 secs and $60^{\circ} \mathrm{C}$ for $45 \mathrm{sec}$. For $k d r$ West, the cycling conditions were $95^{\circ} \mathrm{C}$ for $10 \mathrm{~min}$ followed by 40 cycles of $92^{\circ} \mathrm{C}$ for $15 \mathrm{sec}$ and $60^{\circ} \mathrm{C}$ for $60 \mathrm{sec}$.

\section{Library preparation and 16S rRNA gene amplicon sequencing Using the 341f (TCGTCGGCAGCGTCAGATGTGTAT AAGAGACAGCCTACGGGNGGCWGCAG) and 805r (GTCTCG TGGGCTCGGAGATGTGTATAAGAG} ACAGGACTACHVGGGTATCTAATCC) primers [26] with Illumina ${ }^{\circledR}$ (San Diego, CA USA) overhang (in bold typeface), the V3- V4 region of the universal bacterial and archaeal 16S rRNA gene was amplified using genomic DNA from $F_{1}$ mosquitoes. Four no-template controls (PCR grade water), along with the six controls from the DNA extraction step-two cross-contamination controls (soil samples), and four blanks-were also processed. The PCR reaction mixture $(25 \mu$ l total volume) comprised 
of $10 \mu \mathrm{l}$ of $2 \times$ KAPAHiFi HotStart Mix (Roche, Switzerland), $5 \mu \mathrm{M}$ each of $341 \mathrm{f}$ and $805 \mathrm{r}$ primers and $5 \mu \mathrm{l}$ of DNA template which was $\geq 20 \mathrm{ng} / \mu \mathrm{l}$. Reactions were conducted using the BIO-RAD T100 thermal cycler with the following cycling conditions: $95^{\circ} \mathrm{C}$ for $3 \mathrm{~min}$ for initial denaturation, followed by 25 cycles of $95^{\circ} \mathrm{C}$ for $30 \mathrm{sec}$, $55^{\circ} \mathrm{C}$ for $30 \mathrm{sec}, 72^{\circ} \mathrm{C}$ for $30 \mathrm{sec}$ and extension at $72^{\circ} \mathrm{C}$ for $5 \mathrm{~min}$. The resulting amplicons of $\sim 460 \mathrm{bps}$ were purified using Agencourt AMPure XP beads (Beckman Coulter Inc., Indianapolis, IN, USA) at $0.87 \times$ sample volume and eluted in $45 \mu \mathrm{L}$ TE buffer. The purified amplicons including those from blank and cross contamination controls were submitted to the Biotechnology Core Facility at the US Centers for Disease Control and Prevention, Atlanta for library preparation and sequencing.

Sequencing libraries were obtained using index PCR. This comprised NEBNext Hig-Fidelity $2 \times$ PCR master mix New England Biolabs Inc., Ipswich, MA), index primers from Nextera XT Index kit v2 set A, B and D; (Illumina, San Diego, CA), and 10-300 ng of each $16 \mathrm{~S}$ rRNA gene amplicon, along with all controls, as template. PCR thermal cycler conditions were set to: $98{ }^{\circ} \mathrm{C}$ for $30 \mathrm{sec}$, followed by 8 cycles of $98^{\circ} \mathrm{C}$ for $10 \mathrm{sec}, 55^{\circ} \mathrm{C}$ and $65^{\circ} \mathrm{C}$ for $30 \mathrm{sec}$ each, followed by a final extension at 65 ${ }^{\circ} \mathrm{C}$ for $5 \mathrm{~min}$. The resulting products were cleaned using Agencourt AMPure XP beads at $1.2 \times$ volume of each library. These were subsequently analysed for size and concentration, normalized and pooled at a final concentration of $2 \mathrm{nM}$. The pool was denatured using Illumina guidelines for loading onto flow cell for cluster generation, and sequencing was performed on an Illumina Miseq using Miseq $2 \times 250$ cycle paired-end sequencing kits. The resulting sequence reads were filtered for read quality, basecalled and demultiplexed using bcl2fastq (v2.19.1).

\section{Sequencing data quality control and generation of amplicon sequence variants (ASV) table}

Resulting raw paired-end sequencing reads were demultiplexed and imported into the Quantitative Insights Into Microbial Ecology (QIIME) 2 pipeline v.2018.11 [27] for analysis. Primers and adapter sequences were removed using the QIIME2 cutadapt plugin v.2018.11.0 [28]. This was followed by quality filtering using the QIIME2 DADA2 plugin v.2018.11.0 to remove any sequencing errors, denoise and dereplicate paired-end sequences, filter out chimeras, and finally generate a frequency table of Amplicon Sequence Variants (ASVs, also referred hereafter as features) [29]. The quality filtering step was achieved using the denoise-paired command with the following parameters; max_ee: 2 , trunc_q: 2 , trim_left_f: 10, trim_left_r: 10, n_reads_learn: 1,000,000 and all other parameters left as default. The resulting frequency table was subsequently filtered to remove features associated with the controls, and those with frequency $<100$ prior to downstream analysis. Following these steps, 36 susceptible and 39 resistant mosquitoes remained, and were used for downstream analysis. The raw sample sequencing reads generated from this project, including those from negative (blank) and cross contamination (soil samples) controls, along with sample metadata, have been deposited in the National Center for Biotechnology Information (NCBI), Sequence Read Archive under the BioProject PRJNA672031.

\section{Microbial community diversity analysis}

Alpha and beta diversity indices [30] were computed and compared between samples with differing resistance phenotypes. Shannon alpha diversity index, a quantitative measure of community richness and evenness, was computed using the q2-diversity plugin. To avoid introducing bias due to unequal sampling depth, prior to alpha diversity analysis, all samples were rarefied to a depth of 100 ASVs per sample (Additional file 1), which was sufficient to capture the typical low microbiota diversity in individual mosquitoes. The Kruskal-Wallis test was used to compare Shannon diversity indices between insecticide resistant and susceptible samples with Benjamini-Hochberg false discovery rate (FDR) corrections.

Bray-Curtis dissimilarity beta diversity index, a quantitative measure used to determine compositional dissimilarity of features between samples, was also computed using the q2-diversity plugin. The Bray-Curtis dissimilarity matrices were computed using both rarefied (to a sampling depth of 100 ASVs per sample as described above) and unrarefied ASVs. Both resulted in significant differences between the microbiota of resistant and susceptible mosquitoes (Additional file 2), thus, ordination outputs of only the latter are presented. Comparisons of the resulting distance matrices between resistant and susceptible samples were performed using permutational multivariate analysis of variance (PERMANOVA) at 999 permutations with Benjamini-Hochberg FDR corrections. Outputs were visualized using phyloseq package [31] in R [32].

\section{Taxonomic annotation of microbial features}

QIIME2 v 2018.11 q2-feature-classifier plugin [33] was used for taxonomic annotation. The Naïve Bayes classifier [34] was pre-trained on 16S SILVA reference (99\% identity) database v.128 [35]. Using the qiime featureclassifier extract-reads command, trimming was done to only target the V3-V4 region of the 16S rRNA gene ( 425 bps length). The qiime feature-table heatmap plugin was subsequently used to visualize the resulting relative abundance of annotated ASVs across samples. 
The plugin's metrics and clustering methods were set to braycurtis and features respectively.

Testing for differentially abundant microbial features between permethrin resistant and susceptible mosquitoes The linear discriminant analysis (LDA) effect size method (LEfSe) [36] was used to identify ASVs that were differentially abundant between resistant and susceptible mosquitoes. Annotated ASVs were converted into abundance tables and uploaded to LEfSe Galaxy v.1.0 (http://hutte nhower.sph.harvard.edu/lefse/). With default parameters, an alpha value of 0.05 was used for both the factorial Kruskal-Wallis and pairwise Wilcoxon tests within LEfSe, and a threshold value of $>2$ was used on the resulting logarithmic LDA score to identify differentially abundant ASVs. The effect sizes of differentially abundant ASVs were visualized as bar plots.

The analysis of composition of microbiome method, ANCOM [37], was used to verify the results obtained from LEfSe. The ANCOM analysis was called using the q-2 composition plugin, with the transform and difference functions set to log_transform and mean_difference, respectively. All other parameters were set to default. The resulting statistic, W, and its default cut off was used to identify differentially abundant features between resistant and susceptible mosquitoes.

\section{Results}

Summary statistics of permethrin resistance phenotypes, $k d r$ mutations and sequencing data

A total of 133 adult $\mathrm{F}_{1}$ female An. gambiae s.s. were tested for resistance to permethrin using $5 \times(107.5 \mu \mathrm{g} / \mathrm{ml})$ the discriminating dose $(21.5 \mu \mathrm{g} / \mathrm{ml})$ of the insecticide, and $52.6 \%$ of the samples tested were found to be susceptible. One hundred of the screened samples (50 resistant and 50 susceptible) were subsequently processed for characterizing the microbiota and $k d r$ allele frequencies. Of all 100 samples, 99\% had the $k d r$ east (Vgsc_1014S) mutation and the remaining one had the $k d r$ west (Vgsc_1014F) gene mutation. This high frequency of $k d r$ east mutation indicated fixation in the mosquito population and thus precluded further correlation analysis between microbial composition and $k d r$ allele frequencies. Microbial community characterization of all 100 samples yielded $4,319,065$ raw sequencing reads, in addition to 5,226 raw reads from blank and cross-contamination controls (Additional file 3). Following sequencing data quality control and subsequent removal of features associated with controls and those with frequency $<100,36$ susceptible and 39 resistant samples remained and were used for downstream analysis.
Microbiota composition differed between permethrin resistant and susceptible An. gambiae s.s

Comparison of Bray-Curtis dissimilarity indices using PERMANOVA, showed significant differences in bacterial composition between permethrin resistant and susceptible mosquitoes (pseudo-F $=2.33, p=0.001$ ). This heterogeneity in microbial community structure associated with insecticide resistance status was further illustrated by principal coordinates analysis (PCoA), in which the microbiota of susceptible samples clustered closely together and away from those of primarily dispersed resistant samples (Fig. 2).

Considering microbial diversity within each group, a Kruskal-Wallis comparison showed no difference in Shannon diversity between the microbiota of permethrin resistant and susceptible mosquitoes $(\mathrm{H}=0.45, p=0.50)$ (Additional file 4).

\section{Anopheles gambiae s.s. from Tulukuyi consisted of sparse but diverse microbial taxa that differed by permethrin susceptibility status}

Taxonomic annotation was performed to the genus level or to the lowest possible taxonomic rank. The relative frequencies of annotated bacterial taxa for each sample are presented in Fig. 3. Overall, ASVs from An. gambiae s.s. microbiota were assigned to 84 bacterial taxa (Additional file 5), and out of these, less than half (36 taxa) were shared between permethrin resistant and susceptible $A n$. gambiae s.s. There were 28 and 20 unique bacterial taxa in permethrin susceptible and resistant samples, respectively (Fig. 3a, Additional file 4). At the genus level, a total of 66 bacterial genera were identified, 29 of which were shared between resistant and susceptible mosquitoes, while 21 and 16 were unique to permethrin susceptible and resistant mosquitoes, respectively (Fig. 3b, Additional file 5).

The most abundant bacterial taxa across all samples were those assigned to Asaia (38.33\%), Enterobacter (7.25\%), Acinetobacter (3.88\%), Klebsiella (3.84\%), an uncharacterized Enterobacteriaceae (3.30\%), and Lysinibacillus (3.27\%), together accounting for more than $55 \%$ of ASVs (Additional file 5). A total of 16 genera were unique to resistant mosquito samples including Lysinibacillus, Thorsellia, Streptococcus and Altererythrobacter, among others (Suppl 4). The six most dominant genera among resistant mosquitoes were Lysinibacillus (13.97\%), Pseudomonas (11.95\%), Acinetobacter (8.54\%), Thorsellia (6.49\%), Asaia (4.23\%) and Bacillus (4.08\%). On the other hand, 21 genera were only found in the susceptible mosquito samples including Marmoricola, Roseomonas, Dyadobacter, Lactococcus, and Myxococcus, among others. Among susceptible mosquitoes, Asaia was the most 


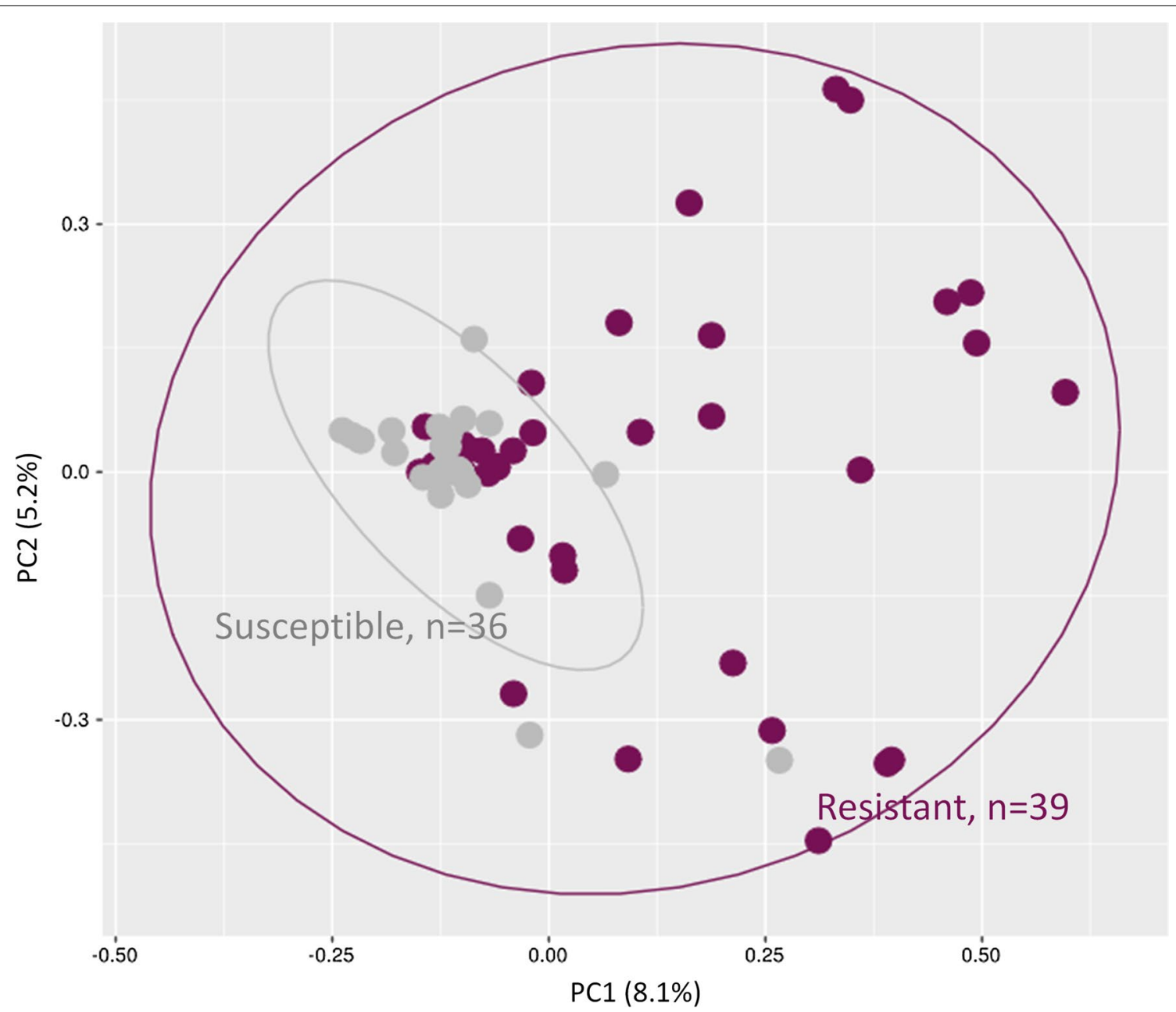

Fig. 2 A Principal Coordinate analysis (PCOA) plot of Bray-Curtis distances between the microbiota of permethrin resistant and susceptible An. gambiae s.s. Each point on the plot represents the microbial composition of a single mosquito. The susceptible samples clustered closely together and away from the primarily dispersed resistant samples. The Bray-Curtis comparison using permutational multivariate analysis of variance (999 permutations) showed a significant difference in microbial composition between resistant and susceptible samples ( $p s e u d o-F=2.33, p=0.001$ )

a

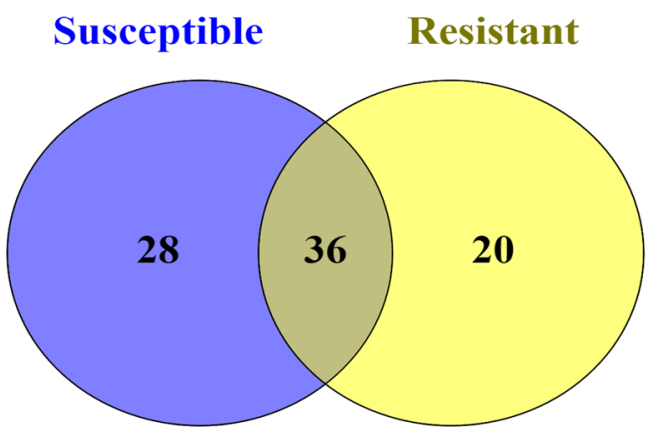

b

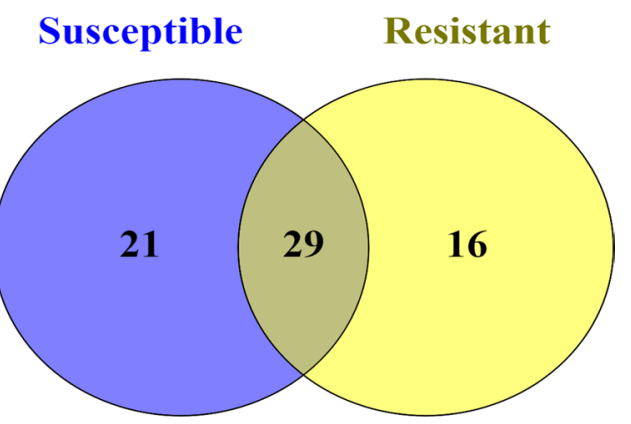

Fig. 3 Venn diagrams showing number of bacterial taxa unique to or shared between 39 permethrin resistant and 36 susceptible mosquitoes. a shows number of bacterial taxa annotated to the genus or lowest possible taxonomic rank, and $\mathbf{b}$ shows number at the genus level 
dominant, with a relative abundance of $48.76 \%$ followed by Enterobacter (9.23\%), Klebsiella (4.41\%), Enterococcus (3.63\%) and Acinetobacter (2.45\%).

A few resistant and susceptible individuals had highly diverse microbiota, with ASVs assigned to between 14 and 37 bacterial taxa (Fig. 4 and Additional file 4). The sample with the highest bacterial diversity was a permethrin susceptible mosquito. Notably, some bacterial taxa were detected more frequently in resistant compared to susceptible mosquitoes. These included the genus Rubrobacter which was detected at low abundance in 20 of the 39 permethrin resistant samples and only in two susceptible mosquito samples, also at low levels of abundance. Similarly, ASVs assigned to unclassified Rhodospirillales (JG37-AG-20) and unclassified Obscuribacteriales were detected in 18 and 9 resistant mosquitoes, respectively, but only in 1 and 4 susceptible mosquitoes, respectively. ASVs assigned to the genera Streptococcus, Thermomonas, Sphingobacterium, Ornithinimicrobium and Lysinibacillus were detected in more permethrin resistant samples compared to the susceptible samples (Fig. 4 and Additional file 4). On the other hand, ASVs annotated as unclassified Enterobacteriaceae were predominant in the susceptible mosquitoes and were detected in 10 of these samples in contrast to only 4 resistant samples.

\section{Differentially abundant bacterial taxa between insecticide resistant and susceptible mosquitoes}

Linear discriminant analysis (LEfSe) also revealed significant differences in microbiota composition between susceptible and resistant mosquitoes. Focusing on the genus level, four bacterial genera, Sphingobacterium, Streptococcus, Lysinibacillus, and Rubrobacter, and an uncultured bacterium were highlighted by LEfSe as more abundant in resistant mosquitoes (Fig. 5). The first three genera were only detected in resistant mosquitoes, while Rubrobacter and the uncultured bacterium were at least three-fold more abundant in resistant compared to susceptible mosquitoes (Fig. 5 and Additional file 5). On the other hand, LEfSe identified only one bacterial genus, Myxococcus, as more abundant in the susceptible samples (Fig. 5); this genus was not detected at all in the resistant samples (Additional file 5). Although more bacterial genera were unique to either resistant or susceptible mosquitoes (Fig. 3b and Additional file 5), LEfSe highlighted those that were present in at least four individuals.

The ANCOM method further corroborated these results. Being more stringent, and not considering features that were unique to either sample category, it identified features assigned to the genus Rubrobacter $(\mathrm{W}=51)$ and unclassified Rhodospirillales (JG37-AG-20) $(\mathrm{W}=63)$ as significantly more abundant in resistant compared to susceptible samples (Additional file 6).

\section{Discussion}

Recently, studies of An. stephensi, An. arabiensis and An. albimanus have shown links between mosquito-associated microbiota and resistance to pyrethroids and organophosphates $[9,10,18,19]$. In this study the microbiota of pyrethroid resistant and susceptible $F_{1}$ progeny of field-derived An. gambiae s.s. were comparatively characterized. Results showed significant differences in microbiota composition between resistant and susceptible mosquitoes with enrichment of different bacterial taxa between resistant and susceptible mosquitoes.

Intense resistance (at $5 \times$ the diagnostic dose) to permethrin was detected, along with high frequency $(99.14 \%)$ of the $k d r$ east allele in the $\mathrm{F}_{1}$ progeny originating from Tulukuyi, Western Kenya. These findings corroborate earlier reports of high pyrethroid resistance in the same area $[6,20]$. Multiple studies from western Kenya have indicated that the high intensity of insecticide resistance may be contributing to mosquito control failure [20,21]. The high frequency of the $k d r$ east allele suggests that the mutation is fixed in this mosquito population. Other studies conducted in western Kenya have also reported the presence of high $k d r$ east allele frequencies which is attributed to the continued use of insecticide-based vector control methods [20, 38-40]. However, results showed that the allele was fixed regardless of resistance phenotype, suggesting that additional mechanisms, such as the overexpression of detoxification enzymes (e.g. cytochrome P450s [41]), are more important than $k d r$ in conferring the intense permethrin resistance detected in the population. The fixation of the $k d r$ east mutation in the population also precluded further analysis of any associations between $k d r$ alleles and the mosquito microbiota. Indeed, a recent study identified no links between the two [42]. The authors thus hypothesize that any microbe-mediated mechanism of insecticide resistance would be largely distinct from the mosquito host's genetics, and likely of a metabolic nature.

Results showed diverse bacterial taxa from individual An. gambiae s.s. samples, a majority of which have previously been identified in Anopheles and other mosquito genera including Aedes aegypti [43-46]. However, less than half of the detected microbial taxa were shared between permethrin resistant and susceptible mosquitoes, suggesting insecticide resistance-related physiological differences that favored different bacterial taxa.

Significant differences in microbiota composition and structure between permethrin resistant and susceptible An. gambiae s.s. were also shown. There is evidence that insecticide detoxifying microbes in agricultural insect pests contribute to insecticide resistance in their hosts $[47,48]$. Recent studies on mosquitoes have also identified insecticide resistance- and/or exposure-driven 


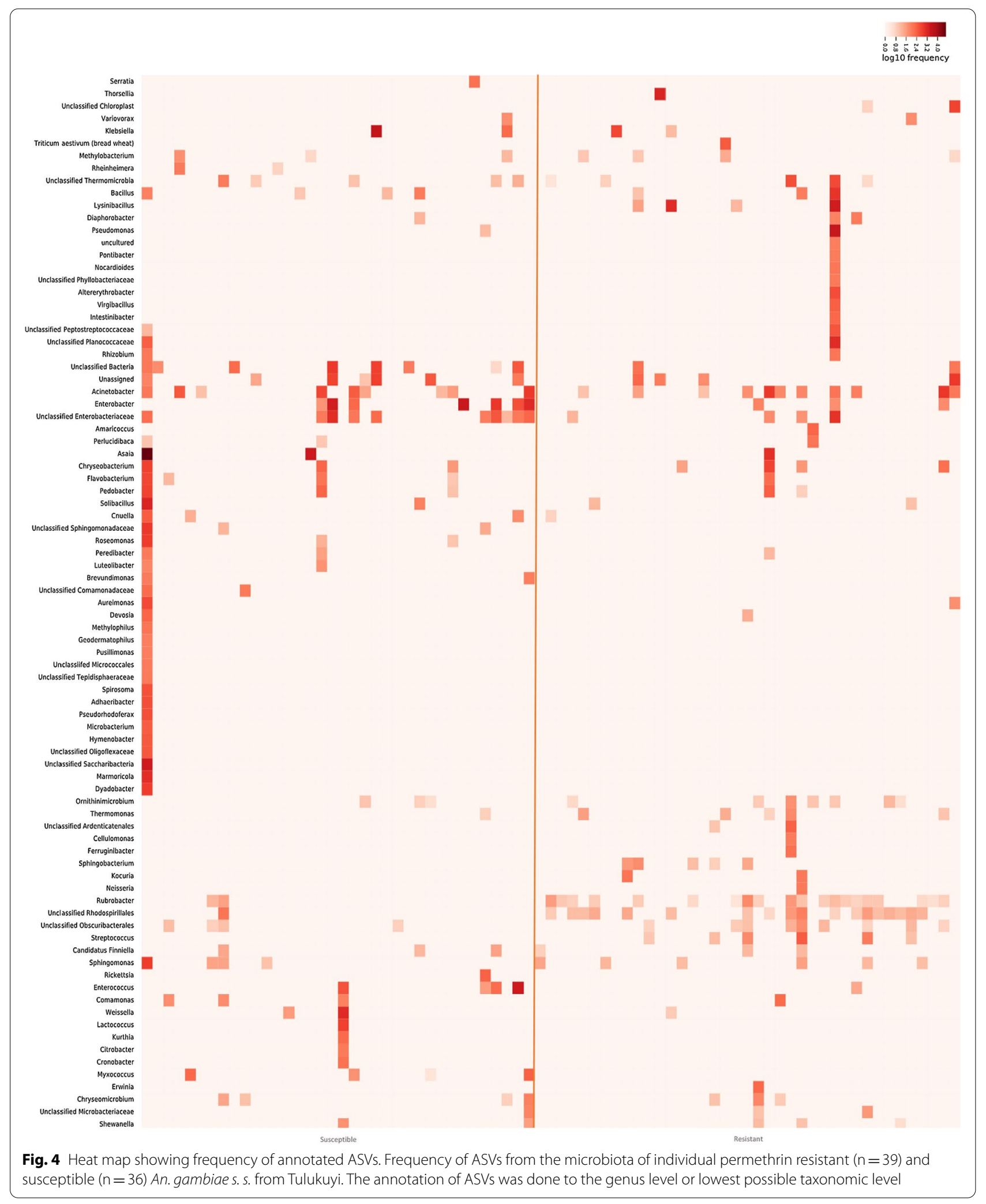




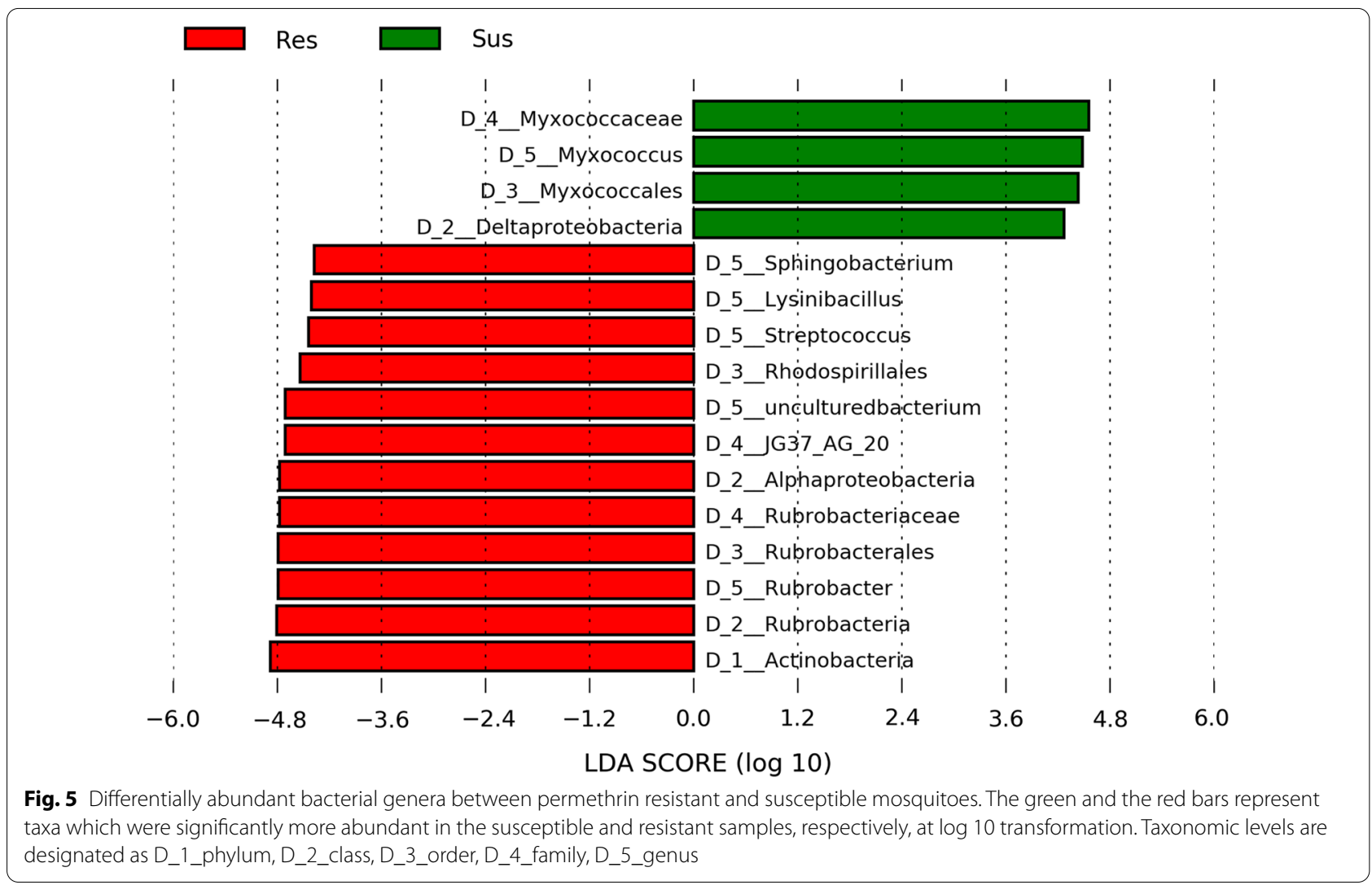

alterations of the host microbiota. In particular, An. albimanus microbiota differed by resistance to fenitrothion and was altered by exposure to different pyrethroids, and Aedes aegypti microbiota differed by resistance to lambda-cyhalothrin $[9,10,49]$. These findings suggest that insecticide resistance in mosquitoes favour and/or is a consequence of the proliferation of certain bacterial taxa, possibly those that can degrade and metabolize insecticides. Recent studies $[9,10]$ identified known insecticide-metabolizing bacterial taxa in An. albimanus that were exposed or resistant to insecticides. Huang et al. [50] and Tang et al. [51] documented that certain microorganisms (considered as potential candidates for bioremediation), including bacteria, degrade pesticides in the soil by breaking them down into smaller compounds, utilizing them as their source of nutrients and making them less toxic to the environment. Some of these microorganisms degrade pesticides to create conducive environments for their survival and not for nutritional requirements [51]. The different taxa present in the resistant versus susceptible mosquitoes, particularly those of resistant mosquitoes, is suggestive of this type of adaptation.
Despite significant differences in microbiota composition and structure (beta diversity), there was no significant difference in alpha (Shannon) diversity between the microbiota of resistant and susceptible mosquitoes. This is suggestive of a homeostatic-controlled number of microbial taxa across individual mosquitoes, with an insecticide resistance-associated perturbation of the type and relative abundance of specific microbial taxa. Mosquitoes used in this study were $F_{1}$ progeny of wild adult females collected from the same location and reared under identical conditions. Except for their permethrin resistance status, which was determined at 2-3 days post adult eclosion, the mosquitoes had identical physiological characteristics. These identical rearing conditions and subsequent uniform physiological characteristics may explain the homogeneity in alpha diversity across samples. On the other hand, the differences in microbial composition associated with their permethrin resistance status provide further evidence of insecticide selection pressure on the mosquito microbiota. Previous studies have shown that a majority of the mosquito microbiota is obtained from mosquito aquatic habitats at the larval stage, and also from food sources as adults [13]. Newly 
emerged adults can also imbibe bacteria along with water from their larval habitats during eclosion or through transstadial transmission [52]. However, other factors such as mosquito physiological status $[11,52,53]$ affect what microbes persist and colonize the mosquitoes following acquisition, and this could explain the insecticide resistance-associated differences in composition despite similar alpha diversity across all individual samples.

Differential abundance testing identified Sphingobacterium, Lysinibacillus, Streptococcus and Rubrobacter as significantly more abundant in resistant mosquitoes and Myxococcus as significantly more abundant in susceptible mosquitoes. The first three genera were only detected in resistant mosquitoes, while Rubrobacter was at least three-fold more abundant in resistant compared to susceptible mosquitoes. In a study conducted by $\mathrm{Hu}$ et al. [54], Lysinibacillus sphaericus was identified as a microbe with the ability to degrade up to $83 \%$ of cyfluthrin (a pyrethroid) after 5 days of incubation by utilizing the insecticide as its source of carbon or nitrogen. In the current study, Lysinibacillus was only detected in resistant mosquitoes, likely as a result of its ability to utilize pyrethroids. Lozano and Dussán [55] also described the potential of Lysinibacillus sphaericus to be used in bioremediation of heavy metals. Sphingobacterium and Streptococcus, also only detected in resistant mosquitoes in this study, are bacterial genera known to degrade pyrethroid insecticides such as cypermethrin [56-58]. Bacteria belonging to the genera Streptococcus and Rubrobacter have been categorized as core microbiota of the digestive system of Anopheles culicifacies [59]. Although not documented for pyrethroid degradation or metabolism, Rubrobacter are known to be thermophilic and extremely resistant to UV thermal and gamma radiations [60]. Other bacterial genera belonging to Actinobacteria, the phylum to which Rubrobacter belongs, have been associated with degradation of insecticides including pyrethroids [61, 62], and the increased relative abundance of Rubrobacter in insecticide resistant mosquitoes could suggest their contribution to resistance. On the other hand, the genus Myxococcus was only detected in susceptible mosquitoes. This bacterial genus is known to be predatory on other bacteria [63], chitinase-producing [64], capable of producing various bioactive antifungal agents [65], and inhibitors of cellular respiration [66]. However, their association with mosquito physiology or insecticide susceptibility has not yet been described. Given what is known about this bacterial genus as highlighted above, it is possible that they could also be toxic to mosquitoes by directly inhibiting host's cellular respiration and/or indirectly preying on other members of the mosquito microbiota that are necessary for host's survival and or insecticide metabolism. Further studies are necessary to elucidate the role of Myxococcus and their secondary metabolites on mosquito physiology, including insecticide susceptibility.

In an aquatic microcosm, it has been demonstrated that insecticides, if used singly or in combination, can reduce microbial diversity and/or induce shifts in microbial community structure [67]. Recent studies have also demonstrated shifts in mosquito microbiota and larval water microbiota that were associated with insecticide exposure $[10,67]$. This indicates that insecticide exposure shapes the microbial composition of mosquitoes and their habitats. This is likely due to the toxic effects of insecticides on some microbes, while at the same time favoring the proliferation of other tolerant microbes as described by Johnsen et al. [68]. It is also possible that in addition to, or rather than selection pressure, the presence of specific insecticide-metabolizing microbes in mosquitoes induce resistance to insecticides and precludes colonization by other microbes. In Ae. aegypti it has been demonstrated that infections with certain microbes precludes colonization by others [69], and that microbial interactions within mosquitoes shape their microbial community [9, 70]. Further research on these microbial networks could shed more light on the role of the mosquito microbiota in insecticide resistance. $[10,67]$.

\section{Conclusions}

In this study, intense permethrin resistance in $\mathrm{F}_{1}$ progeny of field-collected An. gambiae s.s. from Tulukuyi, Bungoma, western Kenya was detected. This was accompanied by a high frequency of $(>99 \%)$ of the $k d r$ east allele, suggesting fixation in the population. These results also show, for the first time, significant differences in microbiota composition between permethrin resistant and susceptible An. gambiae s.s. These findings corroborate results of previous research on other Anopheles species from different geographic locations. The abundance of Rubrobacter, Lysinibacillus, Sphingobacterium and Streptococcus were associated with resistant mosquitoes, while the abundance of Myxococcus was associated with susceptible mosquitoes. The enrichment of these specific bacterial taxa highlights the potential for discovering novel microbial markers of insecticide resistance that could complement existing insecticide resistance surveillance tools. With this increasing evidence of associations between mosquito 
microbiota and insecticide resistance, future work will evaluate the underlying microbial mechanisms of insecticide resistance.

\section{Supplementary Information}

The online version contains supplementary material available at https://doi. org/10.1186/s12936-021-03606-4.

Additional file 1. Comparison of Bray curtis dissimillarity indices between the microbiota of permethrin resistant and susceptible individuals using rarefied and unrarefeid data.

Additional file 2. Shannon diversity indices.

Additional file 3. Taxonomic annotation and frequency of ASVs from permethrin resistant and sucseptible samples.

Additional file 4. ANCOM statistical results.

Additional file 5. Summary statistics of raw sequencing outputs and resulting data following quality control and filtering.

Additional file 6. Shannon alpha diversity index rarefaction curves.

\section{Abbreviations}

ASL: Above sea level; ASVs: Amplicon Sequence Variants; CDC: Centre for Disease Control; FDR: False discovery rate; IRS: Indoor residual spraying; Kdr: Knock Down Resistance gene; KEMRI-CGHR: Kenya Medical Research InstituteCenter for Global Health Research; LDA: Linear Discriminant analysis; LEfSe: Linear discriminant analysis effect size; LLINs: Long Lasting Insecticidal Nets; PCoA: Principal Co-ordinates Analysis; PERMANOVA: Permutational multivariate analysis of variance; QIIME: Quantitative Insights Into Microbial Ecology; rRNA: Ribosomal Ribonucleic acid.

\section{Acknowledgements}

We acknowledge and thank the homeowners in Tulukuyi village for access to their homes during mosquito collections, without which this study would not have been possible; Evans Olang and Duncan Omondi from Kenya Medical Research Institute, for assistance during mosquito collections; Maurice Ombok from Kenya Medical Research Institute for the map of Tulukuyi, and the Biotechnology Core Facility at the US Centers for Disease Control and Prevention for sequencing our samples. We are grateful to the KEMRI Director General for the permission to publish this work.

\section{Authors' contributions}

ND conceptualized and designed the study; EO facilitated and provided laboratory facilities for field work; ND, EO, MS \& AL provided resources for molecular analysis; DO, MK, SO and EE performed mosquito collections, mass rearing and bioassays; $\mathrm{DO}$ and MS performed molecular analysis and sequencing; ND, EMN \& EO supervised the work; DO and ND performed the data analysis and drafted the manuscript; All authors read and approved the final manuscript.

\section{Funding}

This study was supported by funds from Grand Challenges, an initiative of the Bill \& Melinda Gates Foundation (OPP1210769) awarded to the Kenya Medical Research Institute (KEMRI), the US Centers for Disease Control and Prevention (CDC), and the American Society of Tropical Medicine and Hygiene (ASTMH) through the American Committee of Medical Entomology Future Leaders in International Entomology Fellowship awarded to ND. The findings and conclusions in this paper are those of the authors and do not necessarily represent the official position of KEMRI, CDC or ASTMH.

\section{Availability of data and materials}

The raw sample sequencing reads generated from this project, including those from negative (blank) and cross contamination (soil samples) controls, along with sample metadata, have been deposited in the National Center for Biotechnology Information (NCBI), Sequence Read Archive under the BioProject PRJNA672031.

\section{Ethics approval and consent to participate}

This study was approved by the Kenya Medical Research Institute (KEMRI) Ethical Review Board under the scientific steering committee (SSC 2776). Oral consent was obtained from each household head prior to mosquito collections.

\section{Consent for publication}

Not applicable.

\section{Competing interests}

All the authors declare that they have no competing interests.

\section{Author details}

${ }^{1}$ Department of Biochemistry, Microbiology and Biotechnology, Kenyatta University, Nairobi, Kenya. ${ }^{2}$ Entomology Section, Center for Global Health Research, Kenya Medical Research Institute, Kisumu, Kenya. ${ }^{3}$ Biotechnology Core Facility Branch, Division of Scientific Resources, National Center for Emerging \& Zoonotic Infectious Diseases, Centers for Disease Control and Prevention, Atlanta, GA, USA. ${ }^{4}$ Entomology Branch, Division of Parasitic Diseases and Malaria, Center for Global Health, Centers for Diseases Control and Prevention, Atlanta, GA, USA. ${ }^{5}$ Faculty of Science and Technology, Norwegian University of Life Science, Aas, Norway. ${ }^{6}$ Public Health and Epidemiology Department, Nigerian Institute of Medical Research, Lagos, Nigeria.

Received: 23 November 2020 Accepted: 22 January 2021

Published online: 08 February 2021

\section{References}

1. World Health Organization. World Malaria Report 2018. Geneva: World Health Organization; 2018.

2. Ministry of Public Health and Sanitation. The Kenya Malaria Strategy 2009-2018 (Revised 2014). Nairobi: Ministry of Public Health and Sanitation; 2014.

3. Bhatt S, Weiss D, Cameron E, Bisanzio D, Mappin B, Dalrymple U, et al. The effect of malaria control on Plasmodium falciparum in Africa between 2000 and 2015. Nature. 2015;526:207-11.

4. Ochomo E, Bayoh NM, Kamau L, Atieli F, Vulule J, Ouma C, et al. Pyrethroid susceptibility of malaria vectors in four Districts of western Kenya. Parasit Vectors. 2014;7:310.

5. WHO. World Malaria report 2017. Geneva: World Health Organization; 2017.

6. Wanjala CL, Mbugi JP, Ototo E, Gesuge M, Afrane YA, Atieli HE, et al. Pyrethroid and DDT resistance and organophosphate susceptibility among Anopheles spp. mosquitoes, western Kenya. Emerg Infect Dis. 2015:21:2178.

7. Riveron JM, Tchouakui M, Mugenzi L, Menze BD, Chiang M-C, Wondji CS Insecticide resistance in malaria vectors: an update at a global scale. In: Manguin S, Dev V, editors. Towards malaria elimination-a leap forward. London: IntechOpen; 2018.

8. Corbel V, N'Guessan R. Distribution, mechanisms, impact and management of insecticide resistance in malaria vectors: a pragmatic review. In: Manguin S, editor. Anopheles mosquitoes-New insights into malaria vectors. London: IntechOpen; 2013.

9. Dada N, Sheth M, Liebman K, Pinto J, Lenhart A. Whole metagenome sequencing reveals links between mosquito microbiota and insecticide resistance in malaria vectors. Sci Rep. 2018;8:2084.

10. Dada N, Lol JC, Benedict AC, López F, Sheth M, Dzuris N, et al. Pyrethroid exposure alters internal and cuticle surface bacterial communities in Anopheles albimanus. ISME J. 2019;13:2447-64.

11. Minard G, Mavingui P, Moro CV. Diversity and function of bacterial microbiota in the mosquito holobiont. Parasit Vectors. 2013;6:146.

12. Douglas AE. Multiorganismal insects: diversity and function of resident microorganisms. Annu Rev Entomol. 2015;60:17-34.

13. Guégan M, Zouache K, Démichel C, Minard G, Potier P, Mavingui P, et al. The mosquito holobiont: fresh insight into mosquito-microbiota interactions. Microbiome. 2018;6:49.

14. Gonzalez-Ceron L, Santillan F, Rodriguez MH, Mendez D, Hernandez-Avila JE. Bacteria in midguts of field-collected Anopheles albimanus block Plasmodium vivax sporogonic development. J Med Entomol. 2003;40:371-4. 
15. Azambuja P, Garcia ES, Ratcliffe NA. Gut microbiota and parasite transmission by insect vectors. Trends Parasitol. 2005;21:568-72.

16. Boissière A, Tchioffo MT, Bachar D, Abate L, Marie A, Nsango SE, et al. Midgut microbiota of the malaria mosquito vector Anopheles gambiae and interactions with Plasmodium falciparum infection. PLoS Pathog. 2012;8:e1002742.

17. Wang S, Dos-Santos AL, Huang W, Liu KC, Oshaghi MA, Wei G, et al. Driving mosquito refractoriness to Plasmodium falciparum with engineered symbiotic bacteria. Science. 2017;357:1399-402.

18. Barnard K, Jeanrenaud AC, Brooke BD, Oliver SV. The contribution of gut bacteria to insecticide resistance and the life histories of the major malaria vector Anopheles arabiensis (Diptera: Culicidae). Sci Rep. 2019:9:9117.

19. Soltani A, Vatandoost H, Oshaghi MA, Enayati AA, Chavshin AR. The role of midgut symbiotic bacteria in resistance of Anopheles stephensi (Diptera: Culicidae) to organophosphate insecticides. Pathog Glob Health. 2017;111:289-96.

20. Ochomo E, Bayoh M, Brogdon W, Gimnig J, Ouma C, Vulule J, et al. Pyrethroid resistance in Anopheles gambiae ss and Anopheles arabiensis in western Kenya: phenotypic, metabolic and target site characterizations of three populations. Med Vet Entomol. 2013;27:156-64.

21. Omondi S, Mukabana WR, Ochomo E, Muchoki M, Kemei B, Mbogo C, et al. Quantifying the intensity of permethrin insecticide resistance in Anopheles mosquitoes in western Kenya. Parasit Vectors. 2017;10:548.

22. Collins FH, Mendez MA, Rasmussen MO, Mehaffey PC, Besansky NJ, Finnerty V. A ribosomal RNA gene probe differentiates member species of the Anopheles gambiae complex. Am J Trop Med Hyg. 1987;37:37-41.

23. Scott JA, Brogdon WG, Collins FH. Identification of single specimens of the Anopheles gambiae complex by the polymerase chain reaction. Am J Trop Med Hyg. 1993;49:520-9.

24. Centers for Disease Control and Prevention (CDC). Guideline for evaluating insecticide resistance in vectors using the $C D C$ bottle bioassay. Atlanta: CDC; 2010

25. Bass C, Nikou D, Donnelly MJ, Williamson MS, Ranson H, Ball A, et al. Detection of knockdown resistance $(k d r)$ mutations in Anopheles gam biae: a comparison of two new high-throughput assays with existing methods. Malar J. 2007;6:111.

26. Hugerth LW, Wefer HA, Lundin S, Jakobsson HE, Lindberg M, Rodin S, et al. DegePrime, a program for degenerate primer design for broad-taxonomic-range PCR in microbial ecology studies. Appl Environ Microbiol. 2014;80:5116-23.

27. Bolyen E, Rideout JR, Dillon MR, Bokulich NA, Abnet CC, Al-Ghalith GA, et al. Reproducible, interactive, scalable and extensible microbiome data science using QIIME 2. Nat Biotechnol. 2019;37:852-7.

28. Martin M. Cutadapt removes adapter sequences from high-throughput sequencing reads. EMBnet J. 2011;17:10-2.

29. Callahan BJ, McMurdie PJ, Rosen MJ, Han AW, Johnson AJA, Holmes SP. DADA2: high-resolution sample inference from Illumina amplicon data. Nat Methods. 2016;13:581.

30. Alpha. and Beta Diversity Explanations and Commands. https://forum .qiime2.org/t/alpha-and-beta-diversity-explanations-and-comma nds/2282. Accessed 09 Aug 2020.

31. McMurdie PJ, Holmes S. Phyloseq: an R package for reproducible interactive analysis and graphics of microbiome census data. PLoS One. 2013:8:e61217.

32. Crawley MJ. The R Book. 2nd ed. Chichester: John Wiley \& Sons; 2007.

33. Bokulich NA, Kaehler BD, Rideout JR, Dillon M, Bolyen E, Knight R, et al. Optimizing taxonomic classification of marker-gene amplicon sequences with QIIME 2's q2-feature-classifier plugin. Microbiome. 2018;6:90.

34. Zhang H. The Optimality of Naive Bayes (2004). http://citeseerx.ist.psu. edu/viewdoc/summary?doi=10.1.1.483.2183. Accessed 09 Aug 2020.

35. Quast C, Pruesse E, Yilmaz P, Gerken J, Schweer T, Yarza P, et al. The SILVA ribosomal RNA gene database project: improved data processing and web-based tools. Nucleic Acids Res. 2012;41:D590-6.

36. Segata N, Izard J, Waldron L, Gevers D, Miropolsky L, Garrett WS, et al. Metagenomic biomarker discovery and explanation. Genome Biol. 2011;12:R60.

37. Mandal S, Van Treuren W, White RA, Eggesbø M, Knight R, Peddada SD Analysis of composition of microbiomes: a novel method for studying microbial composition. Microb Ecol Health Dis. 2015;26:27663.
38. Mathias DK, Ochomo E, Atieli F, Ombok M, Bayoh MN, Olang G, et al. Spatial and temporal variation in the kdr allele L1014S in Anopheles gambiae s.s. and phenotypic variability in susceptibility to insecticides in Western Kenya. Malar J. 2011;10:10.

39. Wanjala CL, Kweka EJ. Malaria vectors insecticides resistance in different agroecosystems in Western Kenya. Front Public Health. 2018;6:55.

40. Ochomo E, Subramaniam K, Kemei B, Rippon E, Bayoh NM, Kamau L, et al. Presence of the knockdown resistance mutation, Vgsc-1014F in Anopheles gambiae and An. arabiensis in western Kenya. Parasit Vectors. 2015;8:616.

41. David J-P, Ismail HM, Chandor-Proust A, Paine MJ. Role of cytochrome P450s in insecticide resistance: impact on the control of mosquito-borne diseases and use of insecticides on Earth. Philos Trans R Soc Lond B Biol Sci. 2013;368:20120429.

42. Bogale HN, Cannon MV, Keita K, Camara D, Barry Y, Keita M, et al. Relative contributions of various endogenous and exogenous factors to the mosquito microbiota. Parasit Vectors. 2020;13:619.

43. Muturi EJ, Dunlap C, Ramirez JL, Rooney AP, Kim C-H. Host blood-meal source has a strong impact on gut microbiota of Aedes aegypti. FEMS Microbiol Ecol. 2019;95:fiy213.

44. Wang Y, Gilbreath TM III, Kukutla P, Yan G, Xu J. Dynamic gut microbiome across life history of the malaria mosquito Anopheles gambiae in Kenya. PLoS One. 2011;6:e24767.

45. Rani A, Sharma A, Rajagopal R, Adak T, Bhatnagar RK. Bacterial diversity analysis of larvae and adult midgut microflora using culture-dependent and culture-independent methods in lab-reared and field-collected Anopheles stephensi-an Asian malarial vector. BMC Microbiol. 2009:9:96.

46. Villegas LM, Pimenta PFP. Metagenomics, paratransgenesis and the Anopheles microbiome: a portrait of the geographical distribution of the anopheline microbiota based on a meta-analysis of reported taxa. Mem Inst Oswaldo Cruz. 2014;109:672-84.

47. Xia X, Zheng D, Zhong H, Qin B, Gurr GM, Vasseur L, Let. al. DNA sequencing reveals the midgut microbiota of diamondback moth, Plutella xylostella (L.) and a possible relationship with insecticide resistance. PLoS One. 2013;8:e68852

48. van den Bosch TJ, Welte CU. Detoxifying symbionts in agriculturally important pest insects. Microb Biotechnol. 2017;10:531-40.

49. Arévalo-Cortés A, Mejia-Jaramillo AM, Granada Y, Coatsworth H, Lowenberger C, Triana-Chavez O. The midgut microbiota of colombian Aedes aegypti populations with different levels of resistance to the insecticide lambda-cyhalothrin. Insects. 2020;11:584.

50. Huang Y, Xiao L, Li F, Xiao M, Lin D, Long X, et al. Microbial degradation of pesticide residues and an emphasis on the degradation of cypermethrin and 3-phenoxy benzoic acid: a review. Molecules. 2018;23:2313.

51. Tang W, Ji H, Hou X. Research progress of microbial degradation of organophosphorus pesticides. Prog Appl Microbiol. 2018;1:29-35.

52. Coon KL, Vogel KJ, Brown MR, Strand MR. Mosquitoes rely on their gut microbiota for development. Mol Ecol. 2014;23:2727-39.

53. Muturi EJ, Kim C-H, Bara J, Bach EM, Siddappaji MH. Culex pipiens and Culex restuans mosquitoes harbor distinct microbiota dominated by few bacterial taxa. Parasit Vectors. 2016:9:18.

54. Hu GP, Zhao Y, Song FQ, Liu B, Vasseur L, Douglas C, et al. Isolation, identification and cyfluthrin-degrading potential of a novel Lysinibacillus sphaericus strain, FLQ-11-1. Res Microbiol. 2014;165:110-8.

55. Lozano LC, Dussán J. Metal tolerance and larvicidal activity of Lysinibacillus sphaericus. World J Microbiol Biotechnol. 2013;29:1383-9.

56. Zhang J, Zheng J-W, Cho BC, Hwang CY, Fang C, He J, et al. Sphingobacterium wenxiniae sp. nov., a cypermethrin-degrading species from activated sludge. Int J Sys Evol. 2012;62:683-7.

57. Dubey KK, Fulekar M. Investigation of potential rhizospheric isolate for cypermethrin degradation. 3 Biotech. 2013;3:33-43.

58. Shamsuddeen $U$, Inuwa A. Utilization of cypermethrin by bacteria isolated from irrigated soils. Bayero J Pure Appl Sci. 2013;6:19-22.

59. Sharma P, Sharma S, Maurya RK, De TD, Thomas T, Lata S, et al. Salivary glands harbor more diverse microbial communities than gut in Anopheles culicifacies. Parasit Vectors. 2014;7:235.

60. Chanal A, Chapon V, Benzerara K, Barakat M, Christen R, Achouak W, et al. The desert of Tataouine: an extreme environment that hosts a wide diversity of microorganisms and radiotolerant bacteria. Environ Microbiol. 2006;8:514-25 
61. Alvarez A, Saez JM, Costa JSD, Colin VL, Fuentes MS, Cuozzo SA, et al. Actinobacteria: current research and perspectives for bioremediation of pesticides and heavy metals. Chemosphere. 2017;166:41-62.

62. Anandan R, Dharumadurai D, Manogaran GP. An introduction to actinobacteria. In: Dhanasekaran D, Jiang Y, editors. Actinobacteria-basics and biotechnological applications. London: IntechOpen; 2016.

63. Vos M, Velicer GJ. Social conflict in centimeter-and global-scale populations of the bacterium Myxococcus xanthus. Curr Biol. 2009;19:1763-7.

64. Shahbaz U, Yu X. Cloning, isolation, and characterization of novel chitinase-producing bacterial strain UM01 (Myxococcus fulvus). J Genet Eng Biotechnol. 2020;18:1-11.

65. Fatope MO, Al-Kindi SM, Abdulnour AO. Research trends: natural products as pest, microbial disease an tumour control agents. Sultan Qaboos Univ J Sci. 2000;5:55-71.

66. Panter F, Krug D, Müller R. Novel methoxymethacrylate natural products uncovered by statistics-based mining of the Myxococcus fulvus secondary metabolome. ACS Chem Biol. 2018;14:88-98.
67. Muturi EJ, Donthu RK, Fields CJ, Moise IK, Kim C-H. Effect of pesticides on microbial communities in container aquatic habitats. Sci Rep. 2017:7:44565

68. Johnsen K, Jacobsen CS, Torsvik V, Sørensen J. Pesticide effects on bacterial diversity in agricultural soils-a review. Biol Fertil Soils. 2001;33:443-53.

69. Kozlova EV, Hegde S, Roundy CM, Golovko G, Saldana MA, Hart CE, et al. Microbial interactions in the mosquito gut determine Serratia colonization and blood feeding propensity. ISME J. 2021;15:93-108.

70. Hegde S, Khanipov K, Albayrak L, Golovko G, Pimenova M, Saldaña MA, et al. Microbiome interaction networks and community structure from laboratory-reared and field-collected Aedes aegypti, Aedes albopictus, and Culex quinquefasciatus mosquito vectors. Front Microbiol. 2018;9:2160.

\section{Publisher's note}

Springer Nature remains neutral with regard to jurisdictional claims in published maps and institutional affiliations.
Ready to submit your research? Choose BMC and benefit from:

- fast, convenient online submission

- thorough peer review by experienced researchers in your field

- rapid publication on acceptance

- support for research data, including large and complex data types

- gold Open Access which fosters wider collaboration and increased citations

- maximum visibility for your research: over $100 \mathrm{M}$ website views per year

At BMC, research is always in progress.

Learn more biomedcentral.com/submissions 